\title{
Las organizaciones comunitarias de agua potable rural en América Latina:
}

\section{un ejemplo de economía substantiva}

\author{
Chloé Nicolas-Artero \\ Centre de Documentation et de Ressource des Amériques - Université Sorbonne- \\ Nouvelle Paris 3 \\ Email: chloenicolasartero@gmail.com
}

Resumen: En las zonas rurales latinoamericanas, organizaciones comunitarias son responsables de otorgar el servicio de agua potable a sus miembros. A partir del caso chileno, el artículo demuestra que los sistemas comunitarios rurales se fundamentan en una economía substantiva que genera una territorialización y patrimonialización del agua. En un contexto de privatización y mercantilización, los comités se ven afectados o transformados, asimilándose a modos de administración capitalistas privados. Sin embargo estrategias y solidaridades locales, nacionales y transnacionales emergen para defender su forma de producir el agua potable alternativa.

Palabras clave: Organizaciones comunitarias, agua potable, saneamiento, ruralidad, América Latina, Chile.

\section{Community organizations of rural drinking water in Latin America: an example of substantive economy}

Abstract: In Latin American rural areas, community organizations are responsible for granting water service to its members. From the Chilean case, the article shows that rural community systems are based on a substantive economy that generates territorialization and patrimonialization of water. In a context of privatization and commodification, committees are affected or transformed, assimilating to capitalist forms of management. However, local, national and transnational strategies and solidarities emerge to defend their alternative form of produce drinking water.

Keywords: Community organizations, drinking water, sanitation, rurality, Latin America, Chili.

\section{As organizações comunitárias de agua potável rural na América Latina: um exemplo de economia substantiva}

Resumo: Nas zonas rurais latino-americanas, organizações comunitárias são responsáveis de outorgar o serviço de agua potável aos seus membros. A partir do caso chileno, o artigo demostra que os sistemas comunitários rurais fundamentamse numa economia substantiva que gera uma territorialização e 
patrimonialização da agua. Num contexto de privatização e mercantilização, os comités vem-se afetados ou transformados, assimilando-se a modos de administração capitalista privada. No entanto, estratégias e solidariedades locais, nacionais e transnacionais emergem para defender sua forma de produzir a agua potável alternativa.

Palavras-chaves: Organizações comunitárias - agua potável saneamento - ruralidade - América Latina - Chile.

\section{Introducción}

Si históricamente las grandes ciudades latinoamericanas han sido abastecidasen agua potablepor empresas públicas o privadas, las poblaciones rurales han creadosistemas de abastecimientos autónomos a partir de organizaciones comunitarias ya existentes (Mantilla, 2011;Lockwood, 2002). En la actualidad, estas organizaciones comunitarias de agua potable rural se encargan de la administración y del mantenimiento de los sistemas de agua potable rurales y/o del saneamiento (CLOCSAS, 2012). Existen más de 80000 organizaciones formales quienes entregan agua potable a más de 40 millones de personas (Vagliente, 2011). Las Juntas de Agua y Comités de Desarrollo Comunitario de El Salvador, las Juntas Administradores de Acueductos Rurales de Panamá, las Asociaciones de Acueductos Comunales de Costa-Rica, las Juntas administradoras de Agua Potable de Ecuador, o las comunidades de agua de Venezuela forman una alternativa a sistemas municipales y públicos. En México, Honduras, Colombia, Perú, Bolivia, Paraguay, Nicaragua y República Dominicana los sistemas comunitarios, coexisten con sistemas privados, públicos o municipales. En Chile, los comités y cooperativas de Agua Potable Rural (APR) representan una alternativa a la privatización de zonas urbanas. Este artículo demuestra en qué aspectos los sistemas comunitarios constituyen una alternativa de producción del agua potable con respecto a los sistemas públicos, municipales o privados.

Las organizaciones comunitarias son organizaciones sin fines de lucro, conformadas por los habitantes de una localidad rural concentrada, cuyo objetivo es abastecer en agua potable a sus miembros, respectando los criterios de calidad, continuidad y asequibilidad del servicio. La directiva de las organizaciones tiene que captar, potabilizar y administrar el servicio de distribución, y en algunos casos recaudar y tratar las aguas servidas - aunque es mayoritario el uso de un pozo negro o fosas sépticas-. Su particularidad reside en el funcionamiento democrático interno: las decisiones se toman en asamblea, la cual elige a un directorio por un mandato anual. La tarifa del agua, fijada por la asamblea o la directiva, permite cubrir los gastos en inversiones de mantenimiento y extensión de redes. Poseen una concesión de uso de los recursos hídricos, respetando los códigos de agua o leyes de agua nacionales.Los sistemas son creados bajo una iniciativa colectiva local,un programa nacional o una Organización No Guberna- 
mental (ONG). Las formas de gestión son muy variablesen función de las capacidades de funcionamiento internas, las realidades sociales y culturales de la comunidad y sus condiciones de formalidad o informalidad.

Las organizaciones comunitarias de agua adquieren una importancia particular a partir de los años 1990 y 2000 en un contexto de promoción de los principios de descentralización y de participación, y del surgimiento de la «cuestión medioambiental”(Lankao, 2007). La gobernanza "local”“democrática” o "participativa” del agua ha sido objeto de estudios que insisten en las modalidades de coordinación entre actores públicos, privados y comunitarios para definir e implementar la acción pública (Hugon, 2007).

Algunas investigaciones, con un enfoque neo-institucionalista económico (Saavedra, 200; Bravo, 2011)identifican los criterios necesarios a una "buena gobernanza": reducir los costos de instalación del servicio de distribución, la corrupción y respetar el principio de participación.Se interrogan sobre las modalidades de financiamiento y formas de alcanzar economías de escala para mejorar la cobertura de agua potable en zonas rurales o periurbanas (Revollo \&Giovanna; 2010).Se acercan al neo-institucionalismo económico (NIE) (Hall, Rosemary \&Taylor, 1997; Billaudot 2004) adoptandouna lectura economicista por la NIE o IER-,centrándose en el rol de los contratos, los costos de transacción y otros instrumentos incitativos para orientar los comportamientos de los actores supuestamente motivados por una racionalidad instrumental. Adoptan una visión normativa de la gobernanza dando la impresión de que las interacciones entre los actores es pacífica y consensual y que los sistemas se desarrollan de forma uniforme y sincrónica en todas partes(Saavedra,2009).

Otrasinvestigaciones combinan los enfoques neo-institucionalistas económicos, sociológicos e históricos para estudiar los dispositivos institucionales desde una perspectiva dinámica (Ventura, 2011,Domínguez Serrano, 2010). A partir de un enfoque pluri-escalar, analizan las relaciones de poder que existentes entre los actores, pero el análisis de las organizaciones es descriptivo y estático(Gutiérrez-Villalpando, 2013; Córdova, Lourdes, 2007; Cienfuegos, Aguilar, 2012; Vásquez, Alvin, Perpinan, 2012; Graterol, Díaz, 2007). Sin embargo, varios estudios sobre la gestión comunitaria (Jaglin \& Bousquet, 2011, Bakker, 2008, Castellano, Hinestroza, 2002) han señaladola evolución de las relaciones de poder existentes en el seno de las organizaciones decontruyendo así la visión idealizada de la gestión comunitaria del agua. Critican los estudios de E. Ostrom (2010)la cual niega las relaciones de poder en los commons (Bakker, 2008, 2009; Harribey, 2001)entre sus miembros y con los usuarios de la cuenca.

A partir de una perspectiva comprensiva ypluri-escalar el artículoestudia las relaciones económicas, sociales y políticas que se desarrollan en las organizaciones,y con sus interlocutores desde la antropología económica,y así determinar en qué medida revela la existencia de una economía alternativa, a los sistemas públicos y privados.La antropología 
económica estudia la economía con una perspectiva histórica de la economía permitiendosobrepasar los tradicionales debates economicistas centrados sobre la eficiencia del mercado o del Estado (Dardot, 2010). El enfoque substantivista desarrollado por Polanyi (1983) redefine la “economía”, limitada a su sentido formal por lo economistas neoclásicos:

\begin{abstract}
"où la science économique est appréhendée comme la science qui étudie les choix humains face à des ressources limitées sollicitées par des fins multiples et des besoins insatiables". Para Polanyi "Le sens substantif [du terme économique] tire son origine de la dépendance de l'homme par rapport à la nature et à ses semblables pour assurer sa survie. Il renvoie à l'échange entre l'homme et son environnement naturel et social. Cet échange fournit à l'homme des moyens de satisfaire ses besoins matériels".
\end{abstract}

La economía substantiva esta empotrada -embedded- en las reglas sociales, culturales y políticas que rigen ciertas formas de producción y de circulación de los bienes y servicios. La construcción política de una economía de mercado, tras haberse constituido la tierra, el trabajo y la moneda en "mercancías ficticias”,provoca un desempotramiento, es decir, conduce a una autonomización de la economía de los asuntos sociales (Postel \& Sobel, 2010) ${ }^{1}$.

En la esfera del agua, la perspectiva de Polanyi permite demostrar que los procesos de mercantilización y de privatización son el producto de la aplicación de una definición formal de la economía ${ }^{2}$. El artículo analizará el caso chileno, el cual ilustra el proceso de privatización llevado a cabo en la mayoría de las ciudades latinoamericanas en los años 1990 y un proceso de mercantilización de los más acabados del continente (Bauer 1993). Polanyi ha demostrado que el mercado auto-regulado es una construcción social. Éste, - tanto como la constitución de la sociedad entera en su auxiliar - , son constituidos por la aplicación de dispositivos político-jurídicos (Caillé, 2007) que crean un mercado laboral, de la tierra y de la moneda. La construcción política del mercado del aguas en Chile constituye la condición sine qua non de la aparición del mercado auto-regulado en la sociedad neoliberal cuyo desarrollo económico se basa sobre la exportación de recursos naturales (Quiroga 1994). La constitución del agua como una mercancía ficticia comienza en 1977 y alcanza su paroxismo con la proclamación del código de aguas de 1981 (Bauer, 2002), período de dictadura militar encabezada por el General Augusto Pinochet. Si el agua es un bien de uso público, el código refuerza su apropiación privada y consagra su mercantilización ${ }^{3}$ (Bauer, 2015). Los derechos de uso del agua son separados de la propiedad de la tierra y pueden ser vendidos, arrendados o comprados libremente en un mercado, como cualquier otra mercancía ${ }^{4}$ (Bauer, 2002, 2015).Otro aporte de Polanyi es demostrar cómo la decisión de privatizar los sectores en los que el Estado detenía un monopolio de servicio público revela la existencia de una creencia en la superioridad de la economía de mercado y la identificación de una empresa moderna a una empresa capitalista, lo que llama sofismo economicista (Polanyi, 2007). La privatización del servicio de distribución 
del agua revela la existencia de éste sofisma en Chile. La ideología neoliberal retoma la creencia en la superioridad del mercado sobre las otras formas de organización económicas y la modernidad capitalista se convierte en sinónimo de mercado auto-regulado dejando obsoleto el intervencionismo del estado (Moulian, 2002; Sunkel, 2011). El proceso de privatización del servicio comienza por la separación de las funciones de regulación y operativas de la empresa pública SENDOS. Se prosiguió con la búsqueda de la autosuficiencia financiera de la función operativa mediante la aplicación de una tarificación que recobraba los costos de servicio, acompañada de subvenciones al consumo (Jouravlev, 2007). Terminó con la conversión en sociedades anónimas y la creación de los consorcios público-privados ${ }^{5}$.

A pesar de la privatización y mercantilización del agua se mantienen los comités y cooperativas de agua potable rural, creados a partir de 1968 con financiamiento del Banco Interamericano de Desarrollo respondiendo a la iniciativa de la Alianza para el Progreso. Desde 2013, tras 40 años de existencia, se crea la Subdirección de APR del Ministerio de Obras Públicas para aplicar el Programa de APR. En 2016, 1.729 comités y cooperativas de agua potable rural ofrecen el servicio de distribución de agua potable a 1.635.900 habitantes en zonas rurales concentradas (DOH, 2016). En un contexto de mercantilización y privatización del agua que da a ver la preeminencia de la economía formal en la gestión del agua, ¿en qué medida y bajo qué condiciones los comités y cooperativas de agua potable rural revelan la existencia de una economía substantiva en la producción del servicio?

El artículo es el producto de una investigación de tesis de maestría en antropología económica en 2013 realizada en el Institut d'Études Politiques de Toulouse ${ }^{6}$, perseguida en una tesis y un doctorado en geografía. Realicé entrevistas semi-estructuradas a más de treinta comités y cooperativas de APR, elegidas de forma aleatoria, a funcionarios de la Subdirección de APR, de la Dirección de Obras Hidráulicas (DOH) del Ministerio de Obras Públicas (MOP), del Ministerio de Desarrollo Social, de la Superintendencia de Servicios Sanitarios (SISS), representantes de las empresas sanitarias. Participé a las visitas de las Unidades Técnicas (UUTT) a los comités, a reuniones de las organizaciones e inter-institucionales permitiendo hacer observaciones directas.

\section{Economía substantiva: del territorio a la patrimonialización del agua}

\section{Los comités de agua potable rural: un ejemplo de economía substantiva}

Los intercambios económicos de los comités de APR revelan la existencia de la economía substantiva. Ésta permite la producción y la circulación de los medios materiales para satisfacer las necesidades de todos a través del reconocimiento de tres principios de integración económicos instituidos: la reciprocidad - basada en el modelo institucional de la sime- 
tría - la redistribución - alentado por el modelo institucional de la centralidad - y el intercambio - basado sobre el mercado auto-regulado (Polanyi, 1983). La hibridación de estos tres principios económicos en un mismo espaciotiempo equivale a reconocer que la economía esta empotrada en las relaciones sociales, políticas y culturales.

Las relaciones sociales de reciprocidad consisten en intercambios de donación y contra-donación realizados sin ánimo de lucro que unen los grupos o personas por obligaciones reciprocas (Servet, 2007). En los comités los integrantes de la directiva trabajan de forma voluntaria. Los cargos de presidente, secretarios y tesoreros no son remunerados. Si bien algunos comités contratan a un gerente remunerado, la dirección administrativa es ad honorem el cual es motivo de orgullo para los miembros que tienen un perfil de dirigente social de base. Por otra parte, los surcos de las redes fueron construidos por los habitantes de la comunidad durante mingas. Las mingas son prácticas de trabajo colectivo voluntario que beneficiara a la comunidad entera o a parte de ella. Manifiestan la existencia de las relaciones don-contra don dentro de la comunidad. Por otra parte, el principio de intercambio mercantil, se define por la existencia de mercados creadores de precios -price-making markets- pero no lucractivos. Se manifiesta en el financiamiento del sistema (Maucourant et Plocinizsak, 2011): las inversiones se realizan a partir del monto recaudado por las tarifas del agua y los comités de APR no tienen fines de lucro. El monto recaudado genera un fondo de ahorro y un fondo de solidaridad. Éste último manifiesta la existencia del principio económico redistributivo. Las relaciones de distribución, se basan en el principio institucional de la centralidad que permite la colecta, reserva y redistribución de bienes y servicios entre todos los miembros de un grupo o de una sociedad (Polanyi, 1944). En los comités se manifiestan en las ayudas sociales distribuidas a ciertos miembros del comité, tomadas en el fondo de solidaridad constituido a partir de la tarifa del agua. Los fondos tienen distintos objetivos en cada comité de APR: el pago de los gastos mortuorios, la construcción de una biblioteca comunitaria, la entrega de ayudas escolares o de salud.

\section{Conflictos, redefinición de los derechos operativos y membresía}

Las relaciones inter-personales en los comités no son pacíficas: las conflictualidades contribuyen a redefinir los derechos colectivos e individuales del agua (Boelens, 2008) los cuales modifican la imbricación de los tres principios económicos de forma específica y evolutiva a cada comité. La tipología de derechos de agua desarrollada por Boelens (2008) para las organizaciones de regantes permite distinguir los derechos existentes dentro de los comités de APR. El contenido de cada derecho es diferente en cada comité de APR dependiendo de las condiciones físicas y socioculturales. 


\begin{tabular}{|c|c|c|}
\hline Comité & \multicolumn{2}{|c|}{ Miembros del comité } \\
\hline Derecho colectivo & Derechos operativos & $\begin{array}{c}\text { Derecho de participar en la toma de } \\
\text { decisiones colectivas }\end{array}$ \\
\hline \multirow[t]{3}{*}{$\begin{array}{l}\text { Derecho de uso de } \\
\text { una parte del flujo } \\
\text { del agua }\end{array}$} & $\begin{array}{l}\text { Derecho de uso de la } \\
\text { infraestructura de conducción y } \\
\text { distribución para llevar el agua } \\
\text { hacia una casa }\end{array}$ & $\begin{array}{l}\text { Derecho de participar en la decisión sobre } \\
\text { la gestión: definir las tarifas, proyectos, } \\
\text { inversiones, reparto del fondo de } \\
\text { solidaridad }\end{array}$ \\
\hline & $\begin{array}{l}\text { Derecho de ser elegido y ocupar } \\
\text { cargos en la organización del } \\
\text { sistema y para ejecutar las } \\
\text { decisiones con respecto a la } \\
\text { gestión del sistema }\end{array}$ & $\begin{array}{l}\text { Derecho de participar en la decisión sobre } \\
\text { la inclusión/exclusión de miembros.: } \\
\text { definir quiénes pueden y quienes no } \\
\text { pueden ser miembros del sistema. }\end{array}$ \\
\hline & & $\begin{array}{l}\text { Derechos de participar en la decisión sobre } \\
\text { el cambio o la ampliación de las redes e } \\
\text { infraestructuras }\end{array}$ \\
\hline
\end{tabular}

Existen conflictos internos, vinculados a las divergencias en cuanto a las decisiones de gestión administrativa, técnica y financiera que se manifiesta durante la asamblea. Por otra parte, conflictos electorales y políticos atraviesan las comunidades en el momento de elegir la directiva ya que los comités constituyen una instancia de socialización y visibilidad importante en el mundo rural actual. Sin embargo, los conflictos más frecuentes están vinculados con los derechos operativos, -derecho al uso de la infraestructura para llevar agua hasta su domicilio y el derecho de ser elegido y ocupar cargos-, y aparecen con la llegada de nuevos habitantes en la comunidad. Si antes del proceso de globalización, el mundo rural podía ser caracterizado por el tipo de actividades que desarrollaban sus habitantes (agricultura familiar por las comunidades indígenas o campesinas) (Lambi, 2003) en la actualidad es fuente de atracción (residencias segundarias, condominios) para clases sociales favorecidas provenientes de zonas urbanas (Arias, 2005; Avila, 2008). Emerge una zona de interfaz entre mundo rural y urbano en dónde cohabitan una pluralidad de actores y se desarrollan nuevas dinámicas sociales, económicas y culturales (Avila, 1996). Estas recalificaciones socio-territoriales conllevan a nuevos habitantes a solicitar el acceso al servicio, sin participar activamente al comité. Por lo general tienen una concepción diferente del servicio, frente a la cual los habitantes endógenos establecen estrategias para impedirles el acceso y evitar modificaciones internas del comité.

“-entonces esa problemática hay que abordarla. Las casas grandes, no es cierto, las personas que se compran una propiedad y vienen a descansar, no es cierto, que se compran una propiedad iy se hacen una piscina! La gente que llega del norte. ¡Estos sistemas no están avalados para eso! ¡Y si tiene la cantidad de agua para avalarlos, hay que cambiar la política con respecto a esto! ¡Esta persona ya no es una persona que quepa en la condición social para la cual el proyecto se generó! ¡Entonces esta persona no puede ser socio! ¿Qué es lo que pasa con esta persona? Que muchas veces es muy instruida, pero que es lo que pasa, esos conocimientos que tiene no los pone para que la comunidad crezca, sino que los pone para su propio 
bienestar. Entonces que pasa, que muchas veces, esta persona, como es influyente y educada, entonces la gente lo elige como presidente y esta persona ¿qué es lo que hace? Aplica la ley, quizás como corresponde, ien desmedro de estos otros! Entonces ya, si la DOH recomienda subir en 10.000 pesos el agua, iyo subo! Entonces, ¿sabes qué? No subamos 10 , subamos 12 , pero estas personas de aquí, ¿qué es lo que hacen? ¿Cierto? E...y esa problemática se está generando, por todos los lados se están creando parcelas de agrado, condominios, porque terrenos antiguamente agrícolas se están transformando en condominios. ¡Esa es una gran problemática! $\mathrm{Y}$ yo pienso que esas personas para mí no pueden ser socios”7

Estos conflictos revelan, además, la existencia de un arraigo territorial vinculado al comité que crea un sentimiento de pertenencia de los habitantes endógenos (Di Méo, 2005).

\section{Territorialización y patrimonialización del agua}

Las tensiones y resistencias vinculadas dan a ver la existencia de un arraigo territorial vinculado de una territorialización del agua, vinculada a una patrimonialización del recurso (Di Méo, 1970). El territorio del agua del comité se define materialmente por la extensión de sus redes, e idealmente por la historia, valores y representaciones compartidas por los habitantes endógenos. Los esfuerzos realizados por la formación del comité, por lograr la regularización de los derechos de agua, por obtener los títulos de propiedad de los terrenos, los esfuerzos físicos y financieros realizados por el mantenimiento del sistema, la lucha por ser integrados al programa y beneficiar de la ayuda del Estado, conllevan a una valoración de los comités de APR por las personas que lo vivieron. Emerge una brecha en las comunidades entre los que comparten esta historia común al origen de un sentimiento de pertenencia y los que no:

"Todo eso se fue modificando, hemos sido, considero yo, que grandes luchadores en cuanto a proyectos, nosotros hemos logrado grandes proyectos que otros comité ¡no han podido lograr, no! ¡Nosotros tenemos dos pozos! Pero también nos tocó lucha y dolor, rabia y sufrimiento $\dot{c}$ cierto? Calor y... hambre llegamos a pasar para poder lograr encontrar el verdadero dueño del terreno, lograr que nos vendiera el terreno, ¡y así po! ¡Y son luchas constantes!

-¡Pero nunca la directiva se dejó vencer! ¡O sea siguió adelante hasta que consiguió el objetivo que era el suyo!

- ¡Pero es poco valorado! Sabe, usted ¡yo creo que el pueblo no valora la lucha de los dirigentes! ¡No! Siempre son mañosos, todos les roban, 'no, está ahí porque está robando', 'no, porque el otro también va a robar'. Entonces jes doloroso escuchar gente con mente sucia! ¡Pero no valora! Lo único que le interesa es abrir la llave, que salga el agua, y ellos se dan el lujo, y no podemos convencer a la gente que todavía crearle conciencia de que el agua es un elemento que se pueda botar, que el día de mañana se puede terminar, iy no! 
Riegan calles, plantas, los autos, manguerean. Y si usted va a decirle, ‘ ¡eee cuide el agua o disminuya!' Y van a decir 'iy no, para eso la pago!'¿Ve? Entonces... hay un hay un...un contraste y una barrera, yo considero acá en Calingasta, no sé en otros pueblos, no he tenido la oportunidad de conversar con los dirigentes porque he estado alejada también de... Todas estas cosas, pero yo considero que no toman conciencia.

-Entonces (...) ¡ es doloroso! El esfuerzo que han hecho los dirigentes e imagínese, nosotros, no solamente nosotros...yo tengo aquí más de 13 años como dirigente. Pero los que estuvieron antes, esta lucha, ¡si esta gente no hubiera tenido esa idea visionaria, no hubiéramos tenido y no hubiera existido este pueblo! Si no hubiera sido un peladero! (...)

-Entonces, no valoran en absoluto, eh acá, bueno ya la mayoría, de la calle de 19 de septiembre, son los verdaderos socios que existieron en ese tiempo, pero ya los demás no, porque las poblaciones que hubieron, las poblaciones de los socios, llegan y ise instalan con todo! Entonces ¡no han formado la liga de los luchadores!”8

Esta historia común, que excede la del agua puesto que se sobrepone a formas de socialización tradicionales (futbol, escuela, junta de vecinos, comités de madres), refuerza un arraigo territorial y una identidad local

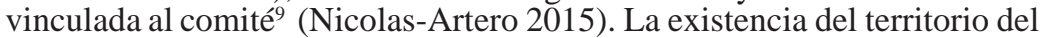
agua se manifiesta en el rechazo de unir varios sistemas cuyas redes se sobreponen - debido a su extensión o su desplazamiento forzado tras la construcción de un embalse. ${ }^{10}$

La territorialización está íntimamente vinculada a la patrimonialización del recurso, entendida como el reconocimiento de un dominio patrimonial que sobrepasa los conflictos de legitimidades entre gestión privada y pública mediante la creación de un mundo común en gestación que da nacimiento una forma de distribución del agua consensual a nivel local (Petit 2009 a.b). La defensa de la patrimonialización en los comités se manifiesta cuando el polo mercantil pasa a ser predominante tras decisiones locales de modernización del sistema, las cuales suelen provenir de proposiciones de habitantes exógenos. Es el caso de la aplicación del artículo 52 bis de la D.F.L. MOP No382/88: ${ }^{11}$

“-Mira acá la finalidad de nosotros del pueblo era lograr este sistema de alcantarillado y poder, aquí se hizo un convenio a través del 52 bis en que Aguas del Valle nos recibe este servicio

-Las aguas servidas

-Nos recibe las aguas servidas,

-Y le hace un tratamiento, todo...todo este sistema jigual que lo...urbano! Pero nosotros lo que pretendíamos y jsiempre nos imaginamos que iba a haber un medidor de caudal! Acá la gente paga caro, carísimo ¡muy caro! Acá Aguas del Valle le cobra individual el recibo y nosotros ¡individual el recibo al agua! Acá Aguas del Valle toma por cada metro cúbico que consume, ¡todo eso baja y 
se lo cobra!

- ¡O sea que una persona que lavó el auto en la calle, y le gustó regar los árboles y todo eso!

-¡Paga!

-Ee... tiene que pagarlo ¡como que aguas se fueron hacia Vicuña!

-Toda el agua que se bota acá, toda esa agua igual la gente la paga.

Entonces yo la encuentro igual absurdo, pero fue la única opción que tuvimos! Otra cosa: Aguas del Valle no se hace cargo de la mantención. Aguas del Valle se hace cargo de un sector no más, y de ahí para acá le corresponde al comité’12

Para los miembros de la directiva, la firma del convenio con la empresa sanitaria aparece como un error en cuanto constataron un aumento el precio del agua de forma desmesurada - lucrativa - sin correlación con el servicio prestado. Para los habitantes endógenos el agua no tiene precio, la tarifa sólo debe cubrir los gastos de mantenimiento el cual sólo se puede garantizar a través del comité cuyo objetivo principal es el bienestar de los habitantes. La economía patrimonial del agua que se quiere defender está íntimamente vinculada al territorio del agua de los comités.

\section{Los comités en el contexto de privatización y mercantilización del agua}

\section{¿Un nuevo sofisma económico en contexto de suburbanización?}

\section{Capacitaciones de las UT: un isomorfismo institucional bajo la forma mer- cantil}

Con la proclamación la privatización de los servicios de agua potable, desaparece la empresa pública SENDOS, y por ende, las unidades públicas encargadas de las capacitaciones comunitarias y técnicas ofrecidas a los comités de APR. A partir de esa fecha y hasta el día de hoy los sistemas rurales quedan sin respaldo normativo: el Programa de APR se mantiene en distintas direcciones del MOP financiado directamente por la ley de presupuestos. El D.F.L. MOP No382/88 establece que las empresas concesionarias privadas tendrán que realizar las capacitaciones técnicas y comunitarias mediante la creación de Unidades Técnicas y diseñar los proyectos de mejoramiento de los comités. Las empresas concesionarias privadas firman convenios con la subdirección de APR que establecen las modalidades de intervención de las UUTT en los comités: por lo general dos intervenciones anuales de seguimiento y un porcentaje de intervenciones en situaciones de urgencia. El objetivo es llegar a la auto-financiación y auto-gestión de los comités para reducir la inversión estatal en el mejoramiento y ampliaciones de las redes. Para lograrlo, el seguimiento técnico durante las visitas anuales, tanto cómo las capacitaciones, fomentan principios ligados a modos de gestión capitalistas que conducen a un isomorfismo institucional bajo la forma mercantil (Laville, 2001). Éste proceso significa la transmisión 
de facultades y representaciones que conducen a una predominancia del principio mercantil en los comités en desmedra de los principios de solidaridad y de reciprocidad. Se manifiesta también por la redefinición de la tarifas para llegar a un auto-financiamiento, tanto como en la supresión de los fondos de solidaridad principal objetivo de los trabajadores de las UUTT y de la SISS.

\section{La expansión urbana: hacia el predominio del principio mercantil.}

Las zonas rurales conocen una dinámica de suburbanización caracterizada por un decrecimiento de la densidad poblacional y la formación de ciudades medias de exportación (Daher, 2014). Se forma una concentración urbana alrededor de los comités de APR por flujo residencial espontáneo o la construcción de poblaciones por el Ministerio de vivienda y urbanismo, puesto que el acceso al agua mejora las condiciones de vida. La multiplicación de arranques y la ampliación de redes conducen a una concentración de capitales de fuerte interés para las empresas concesionarias privadas urbanas (Swyngedouw, 2004).

Por una parte, esta concentración urbana provoca problemas de contaminación hídrica o de sanidad en cuanto los pozos y las fosas sépticas se vuelven insuficientes para almacenar las aguas servidas. A pesar de que pueda deberse a un mal manejo de las fosas, la Subdirección de APR y la SUBDERE financian la construcción de plantas de tratamiento y de redes de alcantarillado en zonas rurales. Según ingenieros hidráulicos, las plantas de tratamiento no son adecuadas en sistemas de poca densidad poblacional pero sus recomendaciones no son atendidas y el Proyecto de ley que regula los servicios sanitarios rurales ${ }^{13}$ obligará los comités a garantizar el servicio de saneamiento. Esta situación entrega la posibilidad a las empresas sanitarias cercanas a los comités de APR de hacer nuevos negocios pudiendo realizar economías de escala al prestar un mismo servicio. Frecuentemente la gestión de la planta de tratamiento pone en dificulta al comité puesto que supone un alza de los costos de mantenimiento (inversiones en infraestructuras, laborales) y genera tensiones en la toma de decisiones ${ }^{14}$. En varias ocasiones las empresas ofrecen prestar el servicio mediante el artículo 52 bis. Los comités conectan sus redes de alcantarillado a la planta de tratamiento de la empresa. A parte del aumento de la tarifa que genera, desestabiliza los comités ya que a veces las empresas condicionan el servicio a una conexión a la red de agua potable (Nicolas-Artero, 2015).

Por otra parte, el D.F.L. MOP N³82/88 establece que todas las zonas urbanas son áreas de concesión de las empresas sanitarias privadas: tienen que respetar criterios de distribución específicos (tarifas fijadas por la SISS, control de calidad, tamaño de redes, presión) y son regulados por la SISS. El proceso de concentración urbana entorno a ciudades rurales medias puede conducir a una modificación del plan regulador y una extensión de la zona urbana. Este fenómeno amplía la zona de concesión de distribución del agua potable y saneamiento para empresas privadas. Los comités de APR cuyas redes pasan a localizarse en zona urbana tienen que solicitar y obte- 
ner el derecho de concesión y para ello, competir con empresas privadas especializadas en el sector. Además, pasan a tener que respetar las normas que se aplican para los servicios sanitarios urbanos. Como los servicios rurales no se rigen con los mismos criterios, los comités en zonas urbanas suelen perder la concesión. Este proceso acaba con su existencia y conduce a un alza del precio del agua para los habitantes que reciben el servicio por una nueva empresa concesionaria privada: triunfa el principio mercantil por la privatización.

\section{Entre penuria y mercantilización: "si no hay agua, no hay comité”}

En la mayoría de las cuencas chilenas existe una penuria de agua que genera un aumento de la competencia por el uso del recurso. La gran parte de las cuencas están “cerradas”: en teoría la DGA ya no otorga derechos de agua superficiales ni subterráneos. Esta situación de escasez afecta a los comités de APR en varios sentidos pudiendo conducir desde un aumento de las tarifas de agua a impedir que realicen su objetivo de captación y distribución.

\section{Los comités frente a la escasez}

Los comités pueden conocer una situación grave de penuria cuando el espejo de agua se reduce de forma abrupta impidiendo la captación del recurso para su distribución. Suele deberse al sobreconsumo de los recursos subterráneos en el acuífero y a una reducción de la infiltración del agua por la urbanización, más que de una reducción pluviométrica. El aumento del consumo está vinculado a un crecimiento demográfico o a cambios en los modos de vida afectando el uso doméstico del agua (Nicolas-Artero, 2015). A veces, la situación crítica radica en el hecho que los derechos de aprovechamiento inscritos en los pozos no corresponden a la cantidad de agua necesaria y utilizada, esta los sobrepasa convirtiendo el uso -aunque no sea fiscalizado ${ }^{15}$ - en ilegal. La inscripción de nuevos derechos suele ser compleja si la cuenca esté cerrada: su precio es inasequible para los comités quienes no benefician de una prioridad de uso - inexistente en el Código de aguas chileno. La incapacidad de inscribir derechos también puede estar vinculada a la imposibilidad de comprar un terreno sobre el cual perforar un pozo nuevo para inscribirlos. En zonas periurbanas la especulación sobre la adquisición de terrenos crea un incremento del precio del metro cuadrado que lo convierte en inasequible. No forma parte de las competencias de la subdirección de APR intervenir en estos casos. Cuando los comités no pueden acceder al recurso físicamente o legalmente, las empresas sanitarias privadas situadas a proximidad proponen venderles agua usando el artículo 52 bis. Estos se convierten en nuevos clientes de las empresas, pierden su autonomía y su rol principal de captación y distribución del agua y aumenta el precio para los habitantes.Cabe señalar, que en el caso de que los comités consigan regularizar los derechos de agua o comprar los terrenos para construir un nuevo pozo, los procedimientos y gastos que atraen debilitan las directivas. 


\section{Urgencia climática, ausencia de debate público y negación de libertad.}

Para hacer frente a la sequía, el gobierno chileno ha creado una nueva medida de gestión de los recursos hídricos a nivel de las cuencas: los decretos de escasez ${ }^{16}$. Permiten al gobierno actuar de forma más rápida sin respetar los procedimientos definidos en el Código de aguas, y aumentan los poderes de la Dirección General de Aguas (DGA) para actuar en cuencas afectadas por la sequía en las que se considera que se tiene que actuar con urgencia. La sequía se define por criterios climáticos y no sociales reducción de nivel de pluviometría o aumento de la evaporación- y se mide por la reducción del espejo de aguas de pozos o embalses y del caudal de los ríos. Una consecuencia frecuente de la aplicación del decreto de escasez es el desplazamiento de derechos de aguas en nuevos puntos de captación cuyo uso dura el periodo determinado en el decreto: seis meses, prorrogados indefinidamente por periodos de seis meses. En la cuenca de la Ligua, el decreto ha permitido a la subdirección de APR del MOP la fusión de siete sistemas de APR en uno solo. Frente a la reducción del espejo de aguas de los comités y la insuficiente capacidad de sus pozos para extraer agua más profunda, el MOP financió la construcción de un pozo de mayor profundidad y capacidad de extracción a partir del cual se reparte el agua a los comités de APR, cuyas redes fueron conectadas entre-si. Esta fusión se realizó de forma obligatoria, la urgencia climática legitimó la ausencia de debate y decisión publica por parte de los interesados-afectados atentando contra su voluntad. Esta experiencia aparece como un plan piloto que podría reproducirse entre comités cercanos entre sí, permitiendo una concentración de redes de agua, de población y por consiguiente de capitales que lo convierte digno de interés para las empresas sanitarias privadas.

\section{Estrategias locales, nacionales e internacionales para mantener la economía substantiva}

\section{Resistencias y alternativas locales y nacionales}

\section{Redes locales de auto-formación para sobrepasar desafíos comunes}

En varias cuencas se conforman redes de solidaridad entre comités de APR para hacer frente a las dificultades comunes que enfrentan. Por ejemplo, Asociación Gremial de Servicios de Agua Potable Rural - VI Región (AGRESAP) y la Asociación gremial de los comités de APR de Elqui se constituyen para hacer frente al débil apoyo que juzgan recibir por parte del Estado y de las UUTT. El antiguo presidente de la Asociación gremial de los comités de APR de Elqui establece su diagnóstico:

"Yo te digo, nosotros como sistema, en la parte rectora en este momento, en la DOH... y... ¡la DOH no tiene el personal! ¡No tiene los técnicos suficientes como para poder atender a estos sistemas! Nosotros, ¡sólo en el Elqui no más somos más de 40! Entonces... Yo 
te digo, y Choapa y Limarí y otros cuantos más. Entonces son muchos los sistemas en la región y la DOH no tiene la capacidad para ayudarlos a todos. Entonces ellos llaman a licitación y en general siempre son las sanitarias las que ganan iy tiene que apoyar en la parte técnica y un poco en la parte administrativa! ¡Pero también po! ¡Es con poca falencia de operación! Porque a nosotros nos visitan a todo restar dos veces en el año y eso es poco, sobre todo para los sistemas que no están con la capacidad de dirigentes o de una buena administración. Los sistemas andan mal y hay muchos sistemas que trabajan regularmente y unos muy mal y son pocos los que trabajan bien.”

En su caso, la empresa sanitaria encargada del diseño y de la asistencia técnica, Aguas del Valle, delega a empresas terceras la gestión de ese servicio. Los dirigentes de los comités están insatisfechos con sus intervenciones, no tienen garantía y los costos son elevados. Frente a ello, prefieren organizarse entre comités y crear un fondo de solidaridad, al que cada comité aporta en proporción a la cantidad de arranques, para apoyarse los unos a los otros - sistema a veces contestado por los mayores contribuyentes.

“-Entonces ¿la idea sería como generar solidaridad entre comités?

-¡Claro! que haya una visión más amplia del problema o sea independiente de que sea que aquí hay un pozo, hay una cantidad de gente, que hay otro aquí, ;que se yo! Tenemos que hacer un macro, porque es la unión hace la fuerza (...)

- ¿Y de qué sirve la asociación gremial?

-¡Capacitación! ¡Necesitamos infraestructura de laboratorios, convenios con laboratorios! ¡Con insumos! Si, estos convenios con estudios de impacto ambiental, cuando se genera una nueva instancia, ¿no es cierto? Eeee asesoría respecto a derechos de agua, ¿no es cierto? ¡A la legislación! Cuanto es un derecho de agua, cuando se reducen, según las necesidades. Todas esas cosas, ¡tiene que haber una asesoría legal para todo!”17

Los recursos del fondo permitirían además organizar encuentros para compartir experiencias, actividades que por el momento se hacen entre directores de los comités más cercanos de manera informal. Se utilizarían para comprar material en común y generar así ahorros, al comprar al por mayor, lo que reduciría los costos de los comités. Estas experiencias han dado impulso a la creación de la Federación Nacional de Agua Potable Rural (FENAPRU), quien ha contado con el apoyo nacional de la organización no gubernamental (ONG) transnacional Fundación Avina, la cual juega un rol importante de apoyo a la gestión comunitaria del agua en Chile y en América Latina.

\section{Capacitaciones ofrecidas por organizaciones nacionales}

Siete organizaciones cuyo objetivo es la capacitación de las cooperativas y los comités de APR en el país fueron inventariadas tras la 
investigación: Federación Nacional de Cooperativas de Servicios Sanitarios (FESAN Ltda.), Instituto Chileno de Educación Cooperativa (ICECOOP), Fundación AVINA, TOHL y FENAPRU. Si cada una de ellas, basadas en Santiago de Chile, dice actuar para la totalidad de los comités del país sus actividades suelen enmarcarse en un contexto local o regional por razones de factibilidad de los proyectos. Su accionar nace de un diagnóstico común: la insuficiente formación y capacitación ofrecida por las UUTT de las empresas sanitarias privadas a las cooperativas y los comités de APR. Las organizaciones quieren reemplazar y suplir este problema creando instancias de capacitación puntuales (conferencias, seminarios, charlas) o planes pilotos con el objetivo de crear instancias de apoyo a largo plazo (escuelas, comités de apoyo). Cada organización se distingue por su estatuto, Fundación AVINA y Tohl son ONGs transnacionales, o por su especialidad, FESAN e ICECOOP trabajan con cooperativas y FENAPRU representa a todos los sistemas de APR del país. Existe cierta competencia o tensiones entre los dirigentes de cada organización, aunque no les impide trabajar en proyectos comunes. Las relaciones con las empresas sanitarias y la DOH son igualmente diferenciadas.

En los últimos años, FESAN y FENAPRU han aplicado el "Programa unificado de fortalecimiento de capacidades” formulado por la Fundación AVINA a nivel regional y aplicado en varios países latinoamericanos, buscando una adaptación en el contexto nacional chileno. Otro proyecto coordinado por la Fundación Avina e implementado por TOHL es la creación de un Centro Comunitario del Agua en la comuna de Rio Hurtado (Región de Coquimbo). Para ello cuentan con el apoyo de la FENAPRU que interviene en los programas de capacitación. Este proyecto ilustra la voluntad de crear Centros de Atención Integral para APR, cuya idea ha sido impulsada a nivel regional latinoamericano por la Confederación Latinoamericana de Organizaciones Comunitarias de Servicios de Agua y Saneamiento (CLOCSAS). Estos centros además de brindar capacitación, pueden brindar asistencia técnica permanente, sirven para canalizar fondos y para brindar otros servicios como la compra de insumos o el acceso a crédito.

\section{La demanda de creación de normas nacionales e internacionales}

\section{La larga lucha por una ley de servicios sanitarios rurales en Chile}

Los comités y cooperativas de APR se encuentran bajo el amparo de ninguna ley específica a los servicios sanitarios rurales y se regulaban bajo la ley 19.418 de Juntas de Vecinos y demás organizaciones comunitarias. La nueva ley sobre servicios sanitarios rurales debiera permitir reconocer las especificidades de los sistemas rurales y protegerlos para asegurar su sostenibilidad. El proyecto de ley fue elaborado y presentado por primera vez al parlamento por la presidente Michelle Bachelet en 2008. Para las 
directivas de los comités, la proclamación de la ley responde a una necesidad local concreta:

"Pero estuve cuando ya hace mucho tiempo atrás que empezamos a hablar de esta ley, porque la ley de estos sistemas, ¡no ayuda mucho! Porque nosotros somos como tierra de nadie, como sistema no somos creíbles, no puedo ir por ejemplo a un banco a pedir plata, y hay muchas otras cosas en la parte laboral, que sin una ley nosotros no podemos operar bien. Pero hoy día nosotros operamos con la ley de juntas de vecinos, pero ya nosotros estamos a la altura de una pequeña empresa y tenemos que manejarnos así como una empresa. Pero con la ley de la junta de vecinos no lo vamos a hacer, ¡no lo vamos a hacer! Entonces estamos a la espera, de que ya esa ley eee... la puedan aceptar y...sería muy favorable para nosotros en muchos aspectos, en muchos aspectos para poder nosotros funcionar como empresa ya.” 18

Para los comités la nueva ley debiera permitir acceder al crédito bancario, regularizar la situación de los trabajadores, permitir mecanismos de sanción en caso de robo, corrupción o falta grave.

FENAPRU ha tenido un papel relevante en la elaboración de la nueva ley. Junto con la Fundación AVINA y Chile Sustentable han realizado un trabajo de lobby para mantener ciertas garantías y defender las especificidades de los comités: los fondos solidarios, la baja tarifa, la gestión democrática. Su mayor inquietud reposaba en que los comités desaparezcan al ser comprados por empresas sanitarias privadas en caso de modificación del plan regulador. Por eso, dos ejes han sido su foco de atención: el que el Estado mantuviera la propiedad de las infraestructuras pensando así impedir ser compradas por las empresas sanitarias y permitir que las capacitaciones técnicas y comunitarias sean realizadas por terceros. Si su accionar puede ser guiado por motivos altruistas la apertura de la licitación para la oferta de capacitación puede crear un mercado de formación, dentro del cual, las organizaciones nacionales pueden tener intereses económicos importantes.

\section{Por una norma de gobernanza internacional común: la "asociatividad”}

A nivel regional en América Latina, la CLOCSAS actúa para valorar las especificidades de la gestión comunitaria y sobrepasar los desafíos comunes con el objetivo de luchar por la patrimonialización del recurso y el derecho humano al agua. ${ }^{19}$ Su creación remonta a 2005, durante el encuentro convocado por la Asociación Hondureña de Juntas Administradoras de Sistemas de Agua, en Honduras, para buscar respuestas a los desafíos del sector. Nació la idea de formar una Federación de Asociaciones Nacionales de Centroamérica y el Caribe de Sistemas de Agua. Dos años después, tuvo lugar en México el Foro de Manejo Comunitario del Agua, que se desarrolla cada dos años, y que reunió en ese año representantes de OCSAS mexicanos, centro-americanos y de algunos países latinoamericanos. En 2010, se 
realizó el Primer Encuentro Latinoamericano de Manejos Comunitarios del Agua en Bolivia que reunió a representantes de todo el continente y tras el cual se creó la plataforma de intercambio de información y conocimiento "WASH rural”. Meses después, en diciembre del 2010, la Fundación AVINA auspició un encuentro en Ecuador "Asociatividades entre Organizaciones Comunitarias Administradoras de Sistemas de Agua Potable y Saneamiento" que dio lugar a la primera reflexión colectiva sobre la "asociatividad", noción que se convertirá en el principio de acción fundamental de la red. En 2011, durante el Segundo Encuentro Latinoamericano de OCSAS en Perú se tomó la decisión de crear la Confederación Latinoamericana de OCSAS.

Según Dupuits (2014), CLOCSAS es un ejemplo de "red transnacional auto-gestionada”: se organiza de manera autónoma y está compuesta por las organizaciones de base directamente concernidas por la problemática levantada ${ }^{20}$. La red se compone de los representantes de las federaciones nacionales como la Red de Organizaciones Sociales y Comunitarias de Gestión del Agua Ecuatorianes (ROSGAE) o la FENAPRU en Chile. Fue creada para promover un modelo alternativo de gobernanza del agua - entorno a principios comunitarios - mediante la transformación de las normas de gobernanza nacionales y referenciales internacionales juzgados inadaptados a las realidades nacionales y locales. Según Dupuits (2014) el objetivo de CLOCSAS es crear una norma de gobernanza medioambiental internacional común, llamada “asociatividad”, definida como:

“Un processus institutionnel d'articulation, d'échange, de communication et de coordination entre les OCSAS d'une localité, région, pays ou continent, afin d'apprendre et de renforcer leurs capacités (de gestion, d'influence sur les politiques publiques, d'innovation), de manière durable, orientées vers le but commun de l'accès à l'eau et à l'assainissement pour tous les latinoaméricains".

También significa una voluntad de mantener su identidad vinculada a la gestión comunitaria frente a las presiones de profesionalización e isomorfismo institucional bajo la forma mercantil existente (CLOCSAS).

\section{Conclusión}

Los comités y cooperativas de APR muestran la existencia de una economía substantiva en la producción del servicio de agua potable. Los tres principios económicos instituidos coexisten de manera evolutiva en el seno de cada comité en función de la redefinición de los derechos internos por los miembros. Las negociaciones revelan conflictos vinculados a los desafíos de la suburbanización actual del mundo rural. Lejos de debilitar el sistema, las conflictualidades son indispensable para el mantenimiento de una economía substantiva anclada en un territorio del agua específico definido por la materialidad de sus redes y la memoria colectiva los cuales conllevan a una patrimonialización del agua. Sin embargo, el sofismo eco- 
nómico, como creencia tiende a imponerse en el mundo rural mediante las capacitaciones ofrecidas y los arreglos locales entre empresas sanitarias privadas y los comités, llevando a una prominencia del principio mercantil. A esto se suma las dificultades de administrar el servicio en un contexto de escasez producida por los discursos sobre la sequía y las herramientas legales, los cuales obligan los comités a actuar contra su voluntad y a veces desaparecer. Frente a las dificultades presentadas, los comités se organizan creando redes de auto-formación y reciben apoyo de organizaciones nacionales cuyo objetivo es frenar el isomorfismo institucional bajo la forma mercantil. La lucha por la elaboración de una ley específica a los servicios sanitarios rurales en Chile y por el reconocimiento de las especificidades de las OCLACS en América Latina demuestra una voluntad directa por lo primeros actores concernidos de defender una forma de producir y de relacionarse al agua alternativa. 


\section{Notas}

${ }^{1}$ Se distingue de la definición de M. Granovetter y de la nueva sociología económica para quienes las actividades económicas están empotradas en el seno de redes de relaciones personales y por consecuente, las instituciones económicas emanan de una construcción social y forman redes de personas inmóviles (Laville, 2001).

${ }^{2}$ La mercantilización constituye el proceso de construcción social por el cual el agua se convierte, dentro de las representaciones cognitivas de los individuos, en objeto asimilable a un capital natural al cual se le asigna un precio de mercado. La privatización del agua reenvía al servicio de abastecimiento de agua potable, cuya gestión históricamente realizada por un ente público es transferida a una empresa se tipo capitalista privada.

${ }^{3}$ No crea un mercado de agua pero las condiciones necesarias a su emergencia espontanea. Es reforzado con declaración de cuencas agotadas.

${ }^{4}$ La Dirección General de Aguas entrega de forma gratuita y a perpetuidad derechos de aprovechamiento si se respetan las condiciones de accesibilidad técnica y los procedimientos de constitución legal. Los derechos de aprovechamiento constituidos son regidos por el derecho privado, deben ser inscritos en el Conservador de bienes raíces, y su posesión se ve garantizada por la protección constitucional de la propiedad privada (Bauer, 2015).

5 Una pluralidad de leyes condujeron a la privatización de los servicios sanitarios urbanos: las leyes $n^{\circ} 18.777$ y 18.885 , de 1989 y 1990, que autorizan al estado para desarrollar actividades empresariales en materia de agua potable y alcantarillado y dispone la constitución de sociedades anónimas para tal efecto; la ley general de servicios sanitarios, D.F.L $n^{\circ}$ 382. MOP de 1988; la ley ${ }^{\circ} 18.901$, de 1990, con la cual se creó la superintendencia de servicios sanitarios; la ley ${ }^{\circ} 19.549$ de 1998 , que modifica el régimen jurídico aplicable al sector de los servicios sanitarios.

${ }^{6}$ La tesis fue realizada bajo la dirección de Catherine Baron y el trabajo de campo se realizó en la Comisión Económica para América Latina con el apoyo de Andrei Jouravlev, director de la unidad de recursos naturales e infraestructuras. Las investigaciones fueron proseguidas en una tesis de geografía bajo la dirección de Sébastien Velut en el Institut des Hautes Études sur l'Amérique Latine de la Univeristé Sorbonne-Nouvelle Paris 3 - que dio lugar a una publicación (Nicolas-Artero 2015)- , y en una tesis doctoral preparada actualmente bajo el mismo director.

7 Entrevista al residente de un comité de la IV región (por respeto del anonimato, no se escribirán los nombres de las personas entrevistadas ni de los comités, en la medida de lo posible), 2016

${ }^{8}$ Entrevista al gerente y al presidente de un comité de APR de la IV región, 2016

${ }^{9}$ En un contexto de privatización de los espacios públicos durante las últimas décadas, los comités se han convertido en un lugar de encuentro, de sociabilidad muy importante en las zonas rurales.

${ }^{10}$ Es el caso de dos comités de APR después de la construcción del embalse Puclaro: 
Polis, Revista Latinoamericana, Volumen 15, $N^{\circ}$ 45, 2016

Punta Azul y la Poblada.

11 En virtud del artículo 52 bis del D.F.L. MOP N³82/88, las concesionarias urbanas pueden establecer, construir, mantener y explotar sistemas de agua potable, alcantarillado y tratamiento de aguas servidas en el ámbito rural. (www.siss.gob.cl)

${ }^{12}$ Entrevista al gerente y al presidente de un comité de APR de la IV región, 2016

${ }^{13}$ Proyecto de Ley que Regula los Servicios Sanitarios Rurales (Boletín N6252-09)

${ }^{14}$ A excepción de las grandes cooperativas y comités como por ejmplos la Cooperativa Hospital-Champa, Cooperativa Coopagua Santo Domingo o El Rosal.

15 Las competencias de la Unidad de Fiscalización de las oficinas regionales de la DGA son limitadas (Entrevista Unidad de Fiscalización DGA Coquimbo, 2016)

${ }^{16}$ Véase el artículo 314 inciso 1 del Código de Aguas

${ }^{17}$ Entrevista al presidente de un comité de la IV región, 2016.

${ }^{18}$ Entrevista al presidente de un comité de APR de la VI Región, 2013.

${ }^{19}$ Sus medios de acción son la creación de Congresos anuales, la proclamación del Día mundial de la gestión comunitaria del agua para aumentar la visibilidad internacional de las organizaciones o la aplicación del Programa Unificado de Fortalecimiento de Capacidades creado por la Fundacion AVINA.

${ }^{20}$ Dupuits hace referencia a la obra deGuarnizo,Luis Eduardo etSmith,Michael Peter (2009). Transnationalism from below. Transaction Publishers. 


\section{Bibliografía}

Avila, S. (2005), (coord.) Lo urbano-rural, ¿nuevas expresiones territoriales? UNAM, Cuernavaca.

Bakker, K. (2008), “The Ambiguity of Community: Debating Alternatives to Private-Sector Provision of Urban Water Supply” en Water Alternative.

Bauer, C. J. (2002), Contra corriente Privatización, mercados de agua y el Estado en Chile. Lom ediciones, Santiago.

Bauer, C. (1993), "Los derechos de agua y el mercado: efectos e implicancias del Código de Aguas chileno de 1981”, en Revista de Derecho de Aguas, volumen IV, Santiago.

Bauer, C. (1996), “Los resultados del mercado de aguas en Chile”, en Ambiente y Desarrollo, volumen XII, $N^{\circ} 2$, junio, Santiago.

Bauer, C. (1997), "Bringing water markets down to Earth: the political economy of water rights in Chile, 1976-95”, World Development, volumen $25, N^{\circ} 5$.

Billaudot, B. (2004), “Institutionnalismes, rationalisme et structuralisme en science sociale“,en Économie et institutions, n 4, p. 5-50.

Boelens, R. (2008), "Water rights arenas in the Andes: Upscaling the defence networks to localize water control" en Water Alternatives 1(1): 48-65.

Bravo, H-M., Castro, J-C., Gutierrez, M-A. (2011), “Evaluación de una política fiscal para determinar el nivel óptimo de la inversión en los servicios de agua potable, alcantarillado y saneamiento.” Gestión y Política Pública, vol. XX, núm. 1.

Caille, A., Laville, J-L. (2007), “Actualité de Karl Polanyi”, en Revue du MAUSS, 1 n²9, p. 80-109.

Castellano, A-M., Hinestroza, J. (2002), “Modo de vida, conocimiento local, planificación y estrategia de gestión del agua en la comunidad El Chaparral, Maracaibo-Estado Zulia”, en Revista Venezolana de Análisis de Coyuntura, vol. VIII, núm. 1, enero-junio.

Cienfuegos, M-A., Aguilar, A. (2012), "Política descentralizadora del agua potable”, en Espacios Públicos, vol. 15, núm. 35.

Clocsas, (2012), "La asociatividad como estrategia en la gestión comunitaria del agua en Latinoamérica.” enInforme Clocsas. 
Cordova, G., Lourdes, M. (2007), “Participación ciudadana en las colonias sin agua potable y alcantarillado de Ciudad Juárez, Chihuahua Nóesis”, enRevista de Ciencias Sociales y Humanidades, vol. 16, núm. 32.

Daher, A. (2014), “Territorios de exportación” en Revista EURE- 16, no 48, Santiago.

Dardot, P., Laval, C., (2010) “Du public au commun”, en Revue du MAUSS, $1 \mathrm{n}^{\circ} 35$, p. 111-122, Pars.

Di Méo, G. (1970), “Patrimoine et territoire, une parenté conceptuelle” en Espaces et sociétés: revue critique internationale de l'aménagement, de l'architecture et de l'urbanisation.

Di Méo, G., Pascal B. (2005), L’espace social. Lecture géographique des sociétés. Armand Colin, Paris.

Dominguez Serrano, J. (2010), “El acceso al agua y saneamiento: Un problema de capacidad institucional local. Análisis en el estado de Veracruz”, Gestión y Política Pública, vol. XIX, núm. 2.

Dupuits, E. (2014), “Construire une norme transnationale en réseau : gestion communautaire de l'eau et “associativité “ en Amérique latine”, RITA [en ligne], $\mathrm{N}^{\circ} 7$ : juin 2014.

Graterol, Y., Díaz, B. (2007), “Situación económica y balance social de las cooperativas que participan en la gestión del servicio público de agua potable en el Estado Trujillo, Venezuela Cayapa. ” enRevista Venezolana de Economía Social, vol. 7, núm. 13.

Guarnizo, L. et Smith, M. (2009). Transnationalism from below. Transaction Publishers, New Jersey.

Gutiérrez-Villalpando, V. (2013), “Mujeres y organización social en la gestión del agua para consumo humano y uso doméstico en Berriozábal, Chiapas Liminar.” en Estudios Sociales y Humanísticos, vol. XI, núm. 2.

Hall, P., Rosemary C. R. Taylor, (1997), "La science politique et les trois néo-institutionnalismes.” en Revue française de science politique, Volume 47 Numéro 3 pp. 469-496

Harribey, J-M. (2011), "Le bien commun est une construction sociale. Apports et limites d'Elinor Ostrom” en L'Économie politique, $1 \mathrm{n}^{\circ}$ 49, p. 98-112, Paris.

Hugon, P. (2007), “Vers une nouvelle forme de gouvernance de l'eau en Afrique et en Amérique Latine” en Revue internationale et stratégique, 2 $\mathrm{N}^{\circ} 66$, p. 65-78 
Jaglin, S. (2005), "La participation au service du neoliberalisme ? Les usagers dans les services d'eau en Afrique subsaharienne.” in Bacque Marie-Hélène, Rey Henri, Syntomer Yves, Gestion de proximité et démocratie participative: une perspective comparative, La Découverte, pp.271-291.

Jouravlev, A., Valenzuela, S. (2007), «Servicios urbanos de agua potable y alcantarillado en Chile: factores determinantes del desempeño”, Santiago, Serie DRNI-CEPAL.

Lankao, P.R. (2007), “Descentralización y retiro del Estado: ¿mecanismos parar gestionar sustentablemente el agua?” enRevista Gestión y política pública, Vol. $16 \mathrm{n}^{\circ} 1$, p.29.

Laville, J-L. (2007), L'économie solidaire, une perspective internationale, Hachette Littératures, Paris.

Laville, J-L. (2001), “Les raisons d'être des associations”', en Association, démocratie et société civile. La découverte/M.A.U.S.S/C.R.I.D.A., p. 61141, Paris.

Lockwood, H. (2002), Mecanismos de apoyo institucional para los sistemas rurales de agua potable y saneamiento manejados por las comunidades en América Latina. USAID.

Mantilla, C. (2011), "Políticas públicas para la prestación de los servicios de agua potable y saneamiento en las áreas rurales”, en DRNI-CEPAL, Santiago.

Maucourant, J., Servet, J-M., Tiran, A. (1998), La modernité de Karl Polanyi, L’Harmattan coll, Paris.

Maucourant J., Plociniczak, S. (2011), “Penser l’institution et le marché avec Karl Polanyi” en Revue de la régulation 10 | 2e semestre / Autumn 2011 http://regulation.revues.org/9439

Moulian, T. (2002), Chile Actual Anatomia de un mito. Lom ediciones, Santiago.

Nicolas-Artero, C. (2015), Memorias de agua. Las organizaciones de usuarios frente a la escasez en la cuenca de Chicureo, Colina (1962-2015). En Revista Tiempo Historico, 6, $\mathrm{n}^{\circ}$ 10, Santiago.

Ostrom, E., Baechler, L., Gouvernance des biens communs. Paris, De Boeck, 2010.

Petit, O. (2009), “Introduction “ La “mise en patrimoine” de l'eau : quelques liens utiles” en Mondes en développement, 1 n 145, p. 7-16, Paris. 
Petit, O., Romagny, B. (2009), “La reconnaissance de l'eau comme patrimoine commun : quels enjeux pour l'analyse économique ? “ en Mondes en développement, $1 \mathrm{n}^{\circ}$ 145, p. 29- 54, Paris.

Polanyi, K. (2007), “Le sophisme économique” en Revue du MAUSS. 1 n²9, p.63-79, Paris.

Polanyi, K. (1983), La grande transformation. Editions Gallimard, Paris.

Postel, N., Sobel, R. (2010), “Le concept de “marchandise fictive “, pierre angulaire de l'institutionnalisme de Karl Polanyi ?” en Revue de philosophie économique, 2 Vol. 11, p. 3-35, Paris.

Quiroga Martinez, R. (1994), El tigre sin selva: consecuencias ambientales de la transformación económica de Chile, 1974-1993, Instituto de Ecología Política, Santiago.

Revollo, D., Giovanna, L. (2010), “Análisis de las economías de escala y alcance en los servicios de acueducto y alcantarillado en Colombia”, Revista Desarrollo y Sociedad, Volumen 66, Bogotá

Saavedra, E. (2011), "La difícil tarea de reformar la institucionalidad regulatoria” en Revista de Análisis Económico, 06, Volumen 26, Número 1, p. 3

Servet, J-M. (2007), “Le principe de réciprocité chez Karl Polanyi, contribution à une définition de l'économie solidaire” en Revue Tiers Monde, 2 n 190, p. 255-273, Paris.

Sobel, R. (2007), Penser la marchandisation du monde avec Karl Polanyi in Sobel R. (coord.), Cahiers lillois d'économie et de sociologie. L'Harmattan, Lille.

Sunkel, O. (2011), Dos siglos de cambio y frustración en Chile. Catalonia, Santiago.

Swyngedouw, E. (2004), Social Power and the Urbanization of Water Flows of Power. Oxford University Press, Oxford.

Vagliente P. (2011), Modelos de gobernabilidad democrática para el acceso al agua en América Latina. Fundación AVINA.

Vasquez, J., Alvin, R., Perpinan, A. (2012), “Propuesta de intervención en el sector agua potable para una comunidad rural: caso de sabana de los javieres en Bayaguana, provincia Monte Plata, Republica Dominicana” en Ciencia y Sociedad, vol. XXXVII, núm. 1. 
Ventura, M.L. (2011), “Aspectos institucionales de la gestión del agua en Pachuca, Hidalgo”, en Revista Mexicana de Sociología 73, núm. 3 p: 509537, Mexico.

Recibido: 31.10 .16

Aceptado: 01.12.16 